\title{
Cambio de la Matriz Productiva vía innovación: Caso Ecuador
}

\author{
Change of the Productive Matrix via innovation: Case Ecuador \\ *Galo Macías España ${ }^{1}$ Diana Cadena Miranda ${ }^{1}$, Jenny Maldonado Castro ${ }^{1}$ Maritza \\ Elizabeth Alcívar Saltos ${ }^{2}$ \\ ${ }^{1}$ Universidad Técnica Estatal de Quevedo. Facultad de Ciencias Empresariales, Escuela de \\ Marketing. Campus Ing. Manuel Haz Álvarez, km 1.5 vía Santo Domingo de Los Tsáchilas. \\ ${ }^{2}$ Universidad Técnica Estatal de Babahoyo km 1.5 vía a Valencia. EC.120501. Quevedo, \\ Ecuador. \\ *gmacias@uteq.edu.ec
}

Fecha de recepción: 05/07/2017

Fecha de aceptación: $30 / 07 / 2017$

Publicado: 24/12/2017

\section{Resumen}

El presente artículo discute algunas de las aristas fundamentales que enlazan variables como el crecimiento económico, transformación productiva, cambios estructurales y algunos elementos asociados a la introducción del desarrollo científico técnico en la producción. Mediante un análisis comparado entre Ecuador y la región Latinoamericana los autores refieren los nexos que se logran y las brechas que separan a la nación ecuatoriana en términos relacionales. Denotan estas valoraciones la necesidad de seguir profundizando en el real cambio productivo que el país demanda para distanciarse de las tradicionales dinámicas que han caracterizado el subcontinente y lograr las metas que defiende la nueva política educativa y de innovación tecnológica ecuatoriana. Se intenta corroborar si en la práctica la innovación conduce a la transformación productiva del Ecuador y cuanto se asegura con ello el cambio en el comportamiento crónico de los ciclos de crecimiento económico.

Palabras clave: crecimiento, matriz productiva, innovación, competitividad.

\section{Abstract}


This article discusses some of the fundamental aspects that link variables such as economic growth, productive transformation, structural changes and some elements associated with the introduction of technical scientific development in production. Through a comparative analysis between Ecuador and the Latin American region, the authors refer to the nexuses that are achieved and the gaps that separate the Ecuadorian nation in relational terms. These assessments denote the need to continue to deepen the real productive change that the country demands to distance itself from the traditional dynamics that have characterized the subcontinent and achieve the goals defended by the new educational policy and technological innovation in Ecuador. It is tried to corroborate if in practice innovation leads to the productive transformation of Ecuador and thus ensures the change in the chronic behavior of cycles of economic growth.

Keyword: growth, productive matrix, innovation, competitiveness.

\section{Introducción}

En los momentos actuales se debate con fuerza las formas y requerimientos para que los pueblos latinoamericanos alcancen estadios en su desarrollo en los que sean sostenibles los ritmos de crecimiento basados en una transformación de la matriz productiva de sus economías, según se revisa en el Compendio Ciencia, Tecnología e Innovación del (BID, 2015).

(Pagés, 2010) "Las generalizaciones sobre las principales dimensiones de la ciencia, la tecnología y la innovación en América Latina indican que, en los albores del siglo XXI, las economías de la región tienden a no hacer uso intensivo de la tecnología y a tener un débil desempeño en cuanto a los resultados de la innovación”, en base a esto se puede afirmar que hasta hoy la experiencia general de los países subdesarrollados es que sus urgencias sociales motivadas por años de desatención a la mayoría de sus ciudadanos, ha conducido a la no 
prioridad de un dinamismo económico que se base en modelos estratégicos potenciadores de las riquezas.

Ecuador ha iniciado un cambio de su política doméstica en materia de cambiar su matriz productiva, tal como lo indica la Agenda para la Transformación Productiva de Ecuador 2010-2013 en donde indica que "es necesario superar la matriz primaria exportadora extractiva y dirigirnos hacia un mayor desarrollo tecnológico, una diversificación productiva y una diferenciación en términos de productos, mercados y actores, que nos permitan insertarnos en una economía globalizada y aprovechar sus oportunidades”, y esta variable: la innovación, ha comenzado a jugar un papel mucho más activo, para lo cual se han establecido nexos entre universidades, institutos de investigación, centros de innovación, ministerios vinculados a la producción, entidades financieras y empresas.

Se somete a discusión la función bidireccional que representa el acceso a la innovación como factor de transformación productiva y la disponibilidad de una transformación productiva que facilite la asimilación de la innovación.

Los estudios que se consultan sobre el Ecuador en materia de este binomio (innovación -transformación productiva) por (Global Entrepreneurship Monitor, 2014) . Los estudios realizados por (BID, 2015), entre otros, no alcanzan a mostrar completamente si existen reservas no explotadas en materia de incrementar las correlaciones entre estas variables y si pudiera ser necesitarse algún cambio de política económica e institucional para el Ecuador en el propósito de incrementar la relación positiva entre innovación y transformación productiva.

\section{Desarrollo}

Las brechas que se han acumulado de diferencias entre el Norte y el Sur no admiten la posibilidad de mantener conductas que repliquen las mismas prácticas internacionales que antes se utilizaron en el crecimiento económico a escala 
mundial. Cada vez es más evidente la necesidad de romper los ciclos de dinámica a escala planetaria para las economías del Sur.

El convencimiento de que la formación de encadenamientos productivos en industrias de alta tecnología con suficiente valor incorporado es condición indispensable en la materialización de lograr el desarrollo, es una hipótesis que se sostienen desde diversas perspectivas analíticas (Schepkova, 2012). Es considerable la proliferación de trabajos en que se discute sobre la interdependencia entre tecnología y transformación productiva tanto para la actividad industrial como la esfera de los servicios, con énfasis en aquellos servicios financieros, comunicacionales y de apoyo al mundo empresarial (Guerrieri, 2005).

La materialización de grados de innovación demanda inversiones en capital humano, tal como lo defienden (Pérez, Zorrilla, \& Velázquez, 2013), (Priede \& Pereira, 2013). En este último trabajo se concluye que el consenso científico internacional de que el progreso económico será dirigido cada vez más por la innovación (invención y aplicación de nuevas tecnologías). Se defiende la hipótesis de que cada vez más juega un papel decisivo el capital intelectual y social en el incremento de los niveles de desarrollo de aquellos países que han adoptado audazmente mejores ambientes empresariales (Spirkova \& al, 2015). Es imposible disponer de capital humano dotado del conocimiento suficiente para enfrentar el reto de la dinámica económica sostenida sin la intervención de políticas institucionales diversas en las que se enrola la educación superior y tecnológica. Hoy nadie discute que la educación superior se considera fuerza indispensable para desarrollar la sociedad del conocimiento (Santos Virgen, 2012).

Así se produce una defensa justificada en varios autores que hablan de la formación de redes del conocimiento para pasar de regiones atrasadas a regiones adelantadas o de 
vanguardia, destacando dentro de estas posiciones el papel que vienen desempeñando las pequeñas y medianas empresas en el crecimiento vía absorción del conocimiento de cara a elevación de la productividad y eficiencia (Huggins \& Johnston, 2009).

Las evidencias empíricas anuncian que los países latinoamericanos, aunque modifican paulatinamente su conducta dinamizadora, aún no alcanzan de forma sostenida a cambiar su comportamiento y por esto siguen quedando a la exposición de un dinamismo crónico y no renovado de su crecimiento en ritmos, temporalidades y tendencias similares al promedio mundial.

La Figura 1 evidencia que entre los años 2005 y 2014 muy análogas han sido las tendencias y dinámicas de crecimiento del PIB a escala internacional y en la subregión. No interesa destacar que puedan estar siendo algo superior las tasas de crecimiento de América Latina respecto al promedio mundial, sino la similaridad del crecimiento y los ciclos en que estos se están produciendo.

Por otro lado, como en la propia figura se expresa, los grados de estabilidad en estas tasas difieren en detrimento de la subregión latinoamericana y caribeña, toda vez que estas han presentado variaciones cercanas a poco más de $7 \%$ promedio y en este mismo período el promedio mundial refleja menos del $6 \%$ de variabilidad. Brechas con disparidades del dinamismo advierten que la estabilidad en el crecimiento puede llegar a ser tan importante para construir dinámicas sostenibles y lo contrario generar procesos destructivos de los necesarios encadenamientos que las economías requieren para solidificar su crecimiento. 


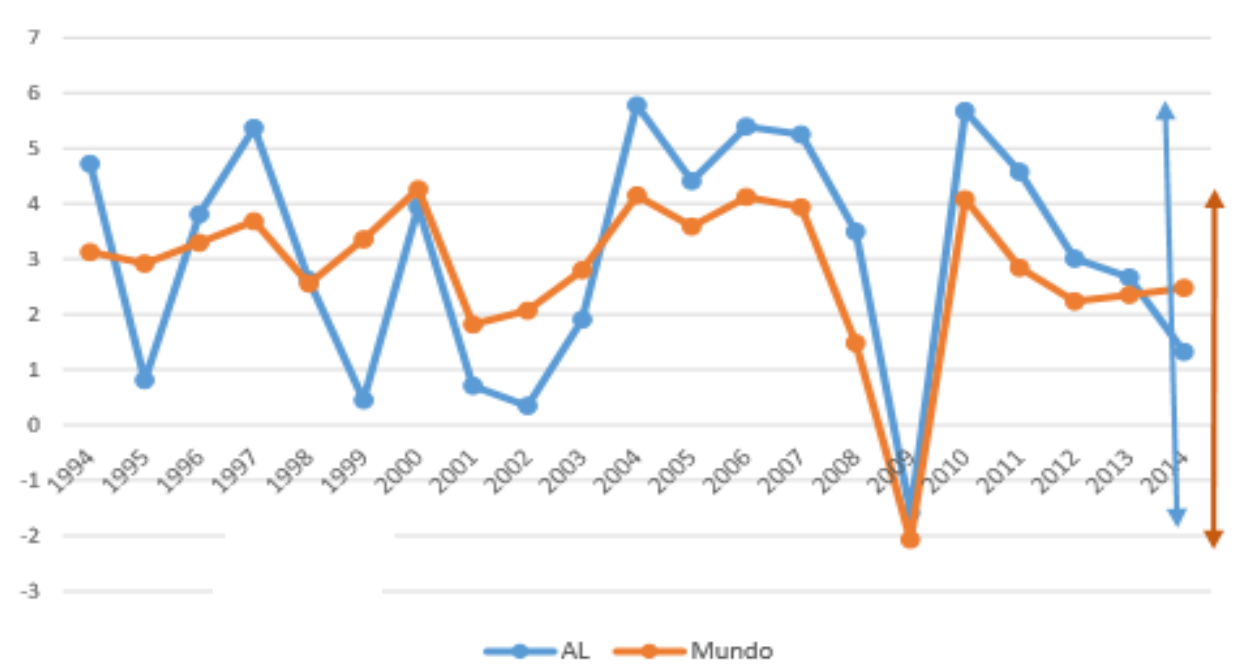

Figura 1. Tasas de crecimiento del PIB del mundo y de los países de América Latina (AL) y el Caribe. Fuente: Elaborado a partir de datos procedentes del World BankData http://databank.worldbank.org/data/home.aspx

Insuficiente ha sido el aprendizaje adoptado por las naciones latinoamericanas y caribeñas en materia de modificar su conducta de crecimiento económico. El caso de Ecuador, en la misma dirección del estudio, muestra el inicio de un proceso de modificación de los ritmos a los que ha venido expandiéndose dentro de esta tendencia dinamizadora (Figura 2), separándose ligeramente en términos de similitud en los ciclos expansivos y recesivos que, aunque aún son insuficientes, determinarán a largo plazo otras causales que en esta discusión científica se intentará abordar. Los procesos de variabilidad de los ritmos de crecimiento del Ecuador respecto al resto de la región no ofrecen diferencias importantes a destacar en el mismo período de análisis, lo cual advierte que todavía hay reservas de estabilidad no logradas en este dinamismo. 


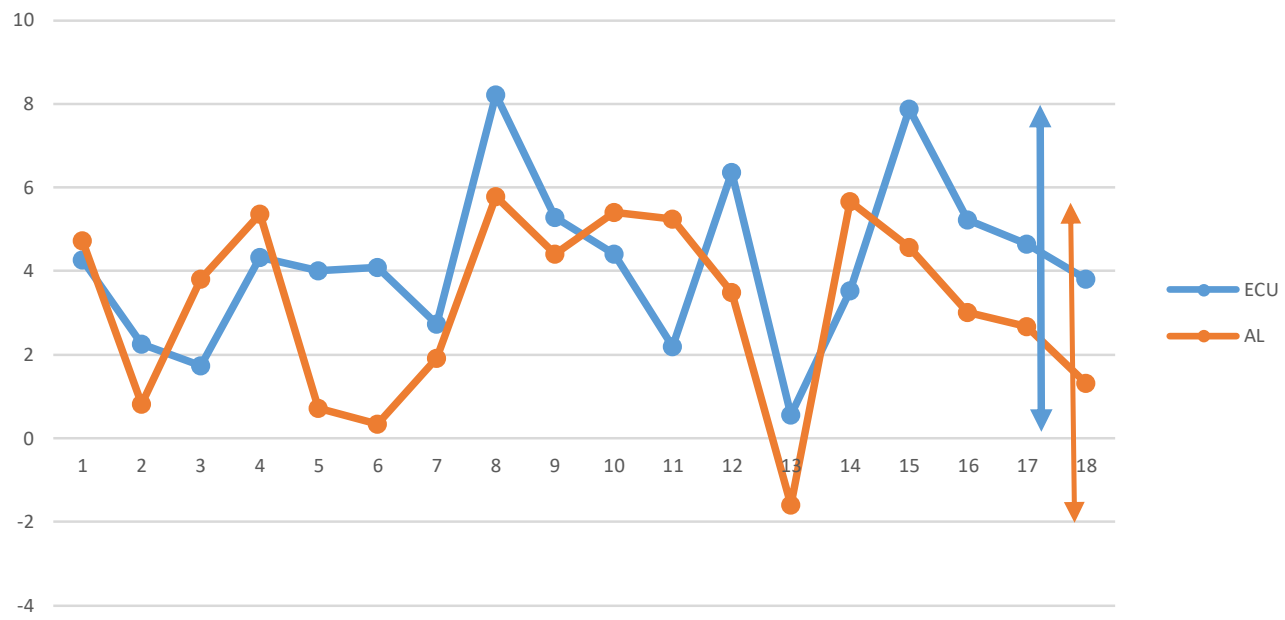

Figura 2. Tasas de crecimiento del PIB de América Latina y el Caribe y Ecuador. Fuente: Ibídem

Lo peor que se está reflejando es que siguen siendo mayores de forma significativa los cambios y oscilaciones que se producen en el dinamismo económico latinoamericano frente al de la economía mundial. Esto se relaciona con las circunstancias particulares de América Latina como lo indica un estudio de la O.C.D.E. de las Perspectivas económicas América Latina 2015 en donde se manifiesta que: "El crecimiento inclusivo requiere de más y mejor innovación. El capital innovación representa un indicador adicional de competencias que mide la capacidad para innovar y diseminar la innovación. El stock de capital de innovación es significativamente inferior en América Latina (13\% del PIB) que en los países de la O.C.D.E. (30\% del PIB).

Este dinamismo económico encuentra una restricción decisiva en los niveles de inversión que las economías producen. Cualquier transformación del crecimiento, aun cuando sea para reproducir los mismos esquemas extensivos o intensivos de crecimiento, demandaría una modificación en los grados de acumulación económica.

Las tasas de inversión (formación bruta de capital/Producto interno bruto) entre los años 1997 y el año 2014 (Figura 3) denotan que los niveles en Ecuador superan sobre todo en los últimos años al promedio de América Latina, oscilando entre un $27 \%$ y un $29 \%$. 


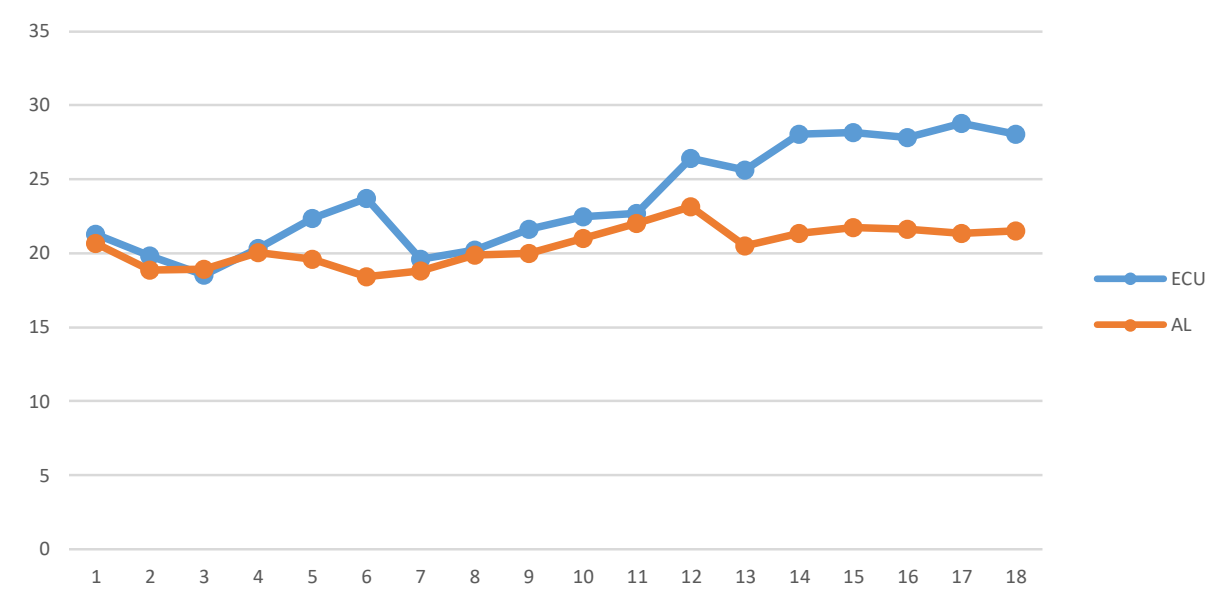

Figura 3. Tasa de formación bruta de capital de América Latina y Ecuador (1997-2014). Fuente: Ibídem

De lo que se trata y se debe atender en el futuro es a los grados de eficiencia con que se materialicen tales niveles de acumulación para mantener un dinamismo económico anti cíclico.

El dinamismo económico diferenciador y fruto de niveles de acumulación suficientes recaba, entre otros ingredientes, de un crecimiento en los encadenamientos productivos y de servicios hacia el interior de sus países.

Un estudio que realizó el Banco Central del Ecuador al clasificar los vínculos visibles provenientes del cálculo de la matriz insumo-producto, tal como aparece en la Figura 4, ofrece importantes observaciones acerca del referido encadenamiento productivo y la estratificación de los sectores económicos en cuatro estadios importantes: "isla", "base", "motor" y "clave". Se estudió el grado de interrelación interna lograda entre los sectores económicos primarios, secundarios y terciarios. 


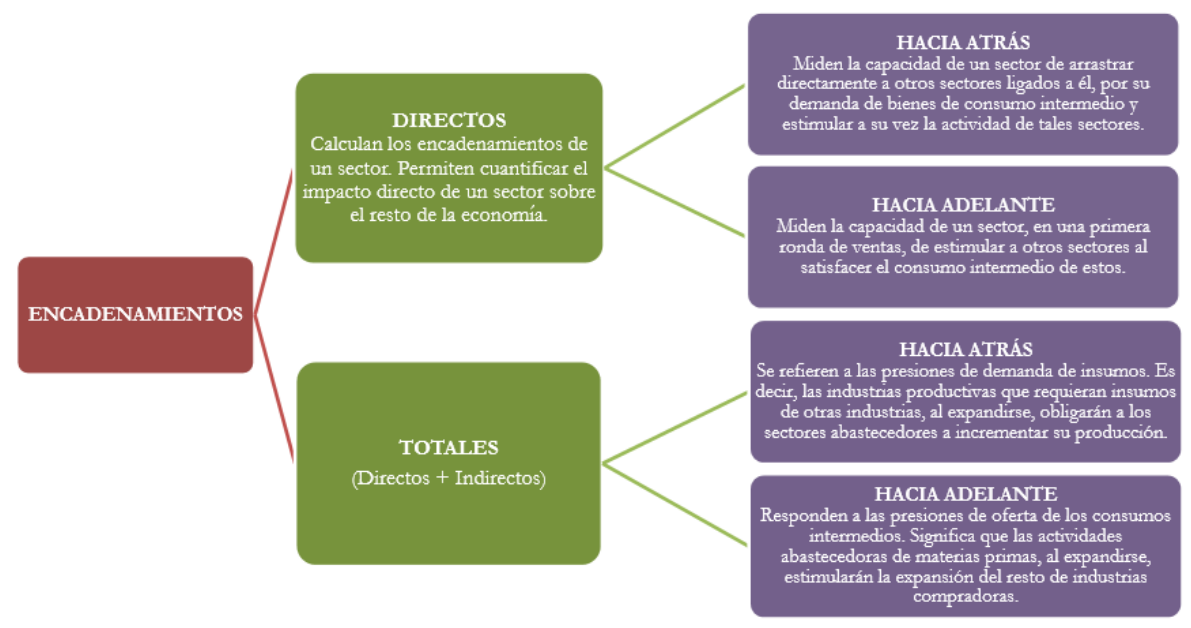

Figura 4. Clasificación de los encadenamientos sectoriales de Ecuador según Matriz InsumoProducto (MIP). Fuente: Banco Central del Ecuador, Marzo 2015. Matrices de insumo producto: simétrica e inversa 2012,

En la figura 4 se aprecia que entre los años 2010 y 2012 de un análisis comparado entre los sectores analizados se constata que los sectores clasificados como "isla" en el 2010 eran el 33,8 \% de los 71 sectores evaluados mientras que en el 2012 la tipología de estos sectores "isla" ascienden al 42,4 \% de los 245 sectores analizados. En estos mismos años los sectores "clave" en el 2010 fueron el 11,3\% en el 2010 y en el 2012 llegaron a ser 9,4 \%.

Potenciar sectores dinamizadores en detrimento de los más ralentizados debería ser la política preferida en condiciones y propósitos de cambio del dinamismo histórico observado. Al hacer una evaluación de los sectores que participan con mayor protagonismo en el crecimiento económico se constata que en Ecuador se mantiene una preeminencia de sectores de poco valor agregado frente a los sectores de mayor dinamización. La agricultura en el caso particular de Ecuador aún representa un peso específico cercano al $10 \%$ dentro de la matriz productiva nacional según cifras del (World Bank Data , 2015).

No obstante la relativa mejor productividad laboral en el sector industria aún permanece el carácter extractivista que ha prevalecido en Ecuador y que recién comienza a modificarse en los momentos actuales. El rol de la renta petrolera ecuatoriana, como se visualiza en la figura 5 manifiesta evidencia de su carácter dependiente de los recursos naturales que 
prevalecía en ciclos económicos anteriores, aún es significativamente superior en relación a los valores promedio latinoamericanos.

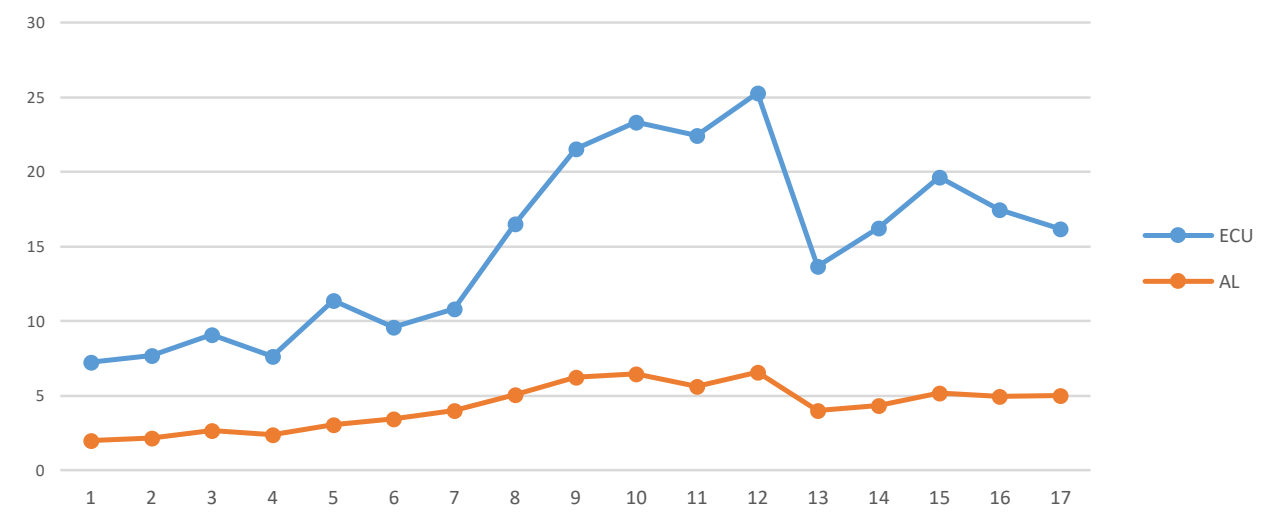

Figura 5. Grado de participación de la renta petrolera en el PIB de Ecuador y América Latina (período 2005 - 2013). Fuente: Ibidem

Degradar el stock de recursos naturales, para aquellos países dotados de recursos atractivos con ventajas naturales en los mercados internacionales, ha conducido a una tradición exportadora de productos básicos, de poco valor agregado en los que la dinámica capitalista de los mercados internacionales ha trasladado la entronización de una cultura extractiva, con la casi ausencia de ciclos transformativos o de servicios.

Observar la estructura de las exportaciones ecuatorianas frente a las de toda América Latina advierte que aún queda camino por recorrer, teniendo preeminencia la presencia de exportaciones de alimentos y sobre todo las exportaciones petroleras con menoscabo de la manufactura. Si a esto se añade la tendencia bajista que se ha observado y se prevé se mantenga según datos del (BID, 2015), en los precios de las materias primas principales es más alarmante esta realidad.

\section{La prioridad: ¿innovación o cambio de la matriz productiva?}

¿Qué política va primero? ¿Cambiar la matriz productiva o acelerar los impulsos innovadores de la economía en su conjunto?

(Pilinkus \& Boguslauskas, 2007) explican que entre los pilares para lograr la competitividad global y materializar altos niveles de productividad y competitividad se ubica 
la innovación junto al desarrollo de las instituciones, la eficiencia de los mercados, el avance infraestructural, la macroeconomía, la educación primaria y la salud, la educación de nivel superior, el aprendizaje tecnológico y la complejización empresarial. Este autor defiende que la innovación es de todos los factores el que asegura los estándares más elevados en el desempeño de las naciones.

(Spirkova \& al, 2015) defienden el nexo que existe entre capital intelectual, capital social e innovación social. Alude que la innovación expresa la disposición de un país u organización a invertir en investigación y desarrollo. Defiende que la innovación social pasa por un ciclo en el que se conjugan tecnologías, procesos, productos nuevos, combinación de ideas, estado jurídico legal propicio y un movimiento social propalador de lo primero.

(Raluka, 2011) expone que la innovación solo se puede convertir en factor clave promotor del desarrollo si está precedida del empleo del conocimiento. La innovación refuerza el papel en la competitividad de empresas, sectores y naciones desplegando estándares de productividad distintivas.

(Priede \& Pereira, 2013) reconocen que la innovación es el despliegue de la invención y desarrollo de nuevas tecnologías. En este trabajo se destacan tres prioridades en la llamada agenda para Europa hacia el 2020 dentro de la cuales resalta el concepto del “crecimiento inteligente", el "crecimiento sostenible" y el "crecimiento inclusivo".

El primero de estos tipos de crecimientos estaría sustentado en el desarrollo de economías basadas en el conocimiento y la innovación. De cualquier forma los otros dos tipos de crecimiento no excluyen el despliegue de nuevos conocimientos.

Una evidente expresión del retraso de las economías latinoamericanas en materia de incorporación de la innovación puede apreciarse en la correlación de la balanza comercial que presentan los niveles de exportación e importación de bienes tecnológicos de la 
información y las comunicaciones incluyendo las telecomunicaciones, audio video, equipamiento relacionado con la computación, otros componentes electrónicos en general, como lo presenta un estudio de la CEPAL en 2015 donde indica que:

En 2014 los valores de las exportaciones y las importaciones de bienes de América Latina presentaron caídas del $2.7 \%$ y el $1 \%$ respectivamente, en comparación con sus niveles del 2013. A nivel de países, las exportaciones de México y algunos países de Centroamérica se beneficiaron de la recuperación que exhibió la economía de los Estados Unidos, país que en varios casos es el destino principal de las ventas al exterior. En los países de América del Sur, en cambio, con algunas excepciones, predominó el efecto del bajo dinamismo de la demanda de bienes por parte de Europa y China, sumado al efecto de la disminución de los precios de exportación, lo que se tradujo en que el valor de las exportaciones se contrajera. En cuanto a las importaciones, también se observó un patrón de diferenciación entre países. En aquellos donde la inversión y el consumo privado experimentaron aumentos, en general también se incrementaron las importaciones, por ejemplo, en Bolivia, Colombia, el Ecuador y varias economías de Centroamérica, entre otros.

Un clásico componente estructural identifica a buena parte de los países latinoamericanos que se dedicaron a apoyar sus desempeños en la renta generada por sus sectores más productivos y mejor posicionados en los mercados internacionales, aunque sus grados de agregación de valor sean poco significativos.

No es posible que se discuta la introducción del factor innovación tecnológica y su impacto sobre el desarrollo sin discutir el lugar que ocupan dentro de aquella las estructuras institucionales y sus políticas asociadas facilitadoras o distorsivas de alcanzar lo primera. (Simmie \& Wood, 2002). 
En realidad, todos los ejemplos históricos de procesos de desarrollo y de crecimiento sostenidos en el tiempo, desde la Revolución Industrial en adelante, se dieron en contextos caracterizados por un conjunto de instituciones diferentes, públicas y privadas que la trayectoria de desarrollo (Ciriaci, 2008).

Se afirma que la innovación se convierte cada vez más en factor detonante del grado de competitividad de las naciones en caso de ubicarse en los sectores estratégicos de estos países. Así ha proliferado con fuerza la defensa de la formación de clúster de alta tecnología (Porter, Newman, \& Roessner, 2009).

(Rivera Vargas, 2014) aborda el rol de la innovación en el desempeño de naciones, firmas y organizaciones para alcanzar un mejor resultado económico. Esta autora desarrolla una demostración de los principales factores que aplican para empresas pequeñas el aprendizaje organizacional y el estilo de liderazgo junto a un desenvolvimiento con una incorporación decisiva de la innovación vía elevación de la competitividad.

En Ecuador dentro del cambio de su política doméstica en materia de economía nacional, la variable: la innovación, ha comenzado a jugar un papel mucho más activo, para lo cual se han establecido nexos entre universidades, instituciones educativas, ministerios vinculados a la producción, entidades financieras y emprendimientos.

Todo un entramado de políticas públicas encaminadas a despertar las necesidades y oportunidades de creación de una cultura del saber se han desarrollado bajo el eje denominado Diálogo de saberes. De aquí se han desplegado varias ideas en calidad de subproyectos. Recientemente ha salido a la luz pública una convocatoria desde la Secretaria Nacional de Educación, Ciencia y tecnología del Ecuador (SENESCYT) acerca de la conformación del proyecto Ingenios, también de la convocatoria de Banco de ideas, o del proyecto CreaCiencia. Solo estos tres casos son pruebas de una proliferación de ambientes que intentan incentivar el desarrollo del espíritu emprendedor desde una visión nacional y 
convertir en hábitos la educación, vida y desenvolvimiento de todos los ciudadanos de la nación.

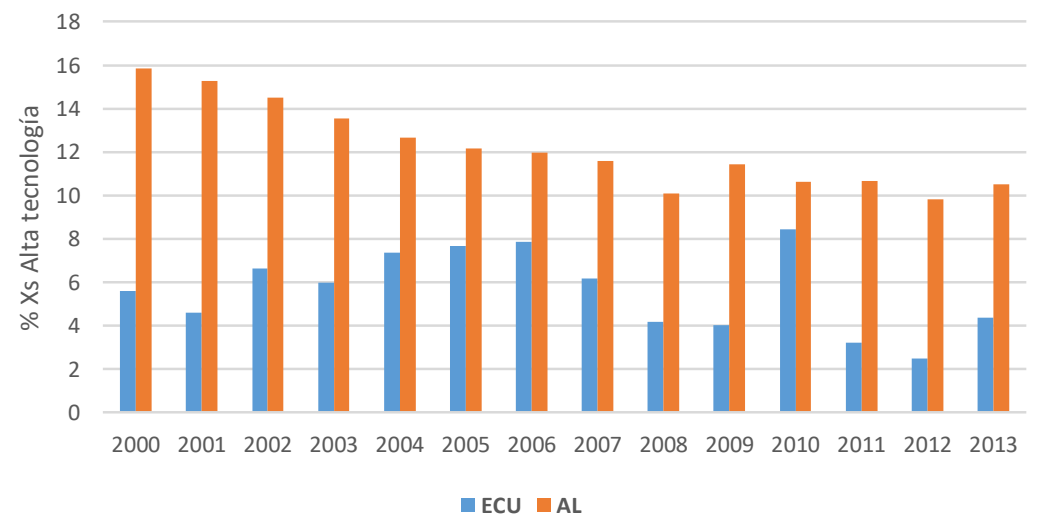

Figura 6. Peso (\%) de exportaciones dentro de la manufactura que incorporan la alta tecnología. Fuente: Ibídem

América Latina (Figura 6) deteriora el nivel de exportaciones de manufactura de alta tecnología entre los años 2000-2013, mientras en el caso de Ecuador se mantiene un proceso que recién incorpora cierto dinamismo aún no estabilizado.

Esta situación genera entre otros factores una desproporción de la protección de la propiedad intelectual en las aplicaciones de los derechos de autor lo cual provoca una pérdida de liquidez para el país. Hoy Ecuador esta una situación más desfavorecida en los flujos de patentes aplicadas de autores no residentes frente a los residentes, cuya razón es extraordinariamente aún más alta que el promedio de América Latina. (Figura 7).

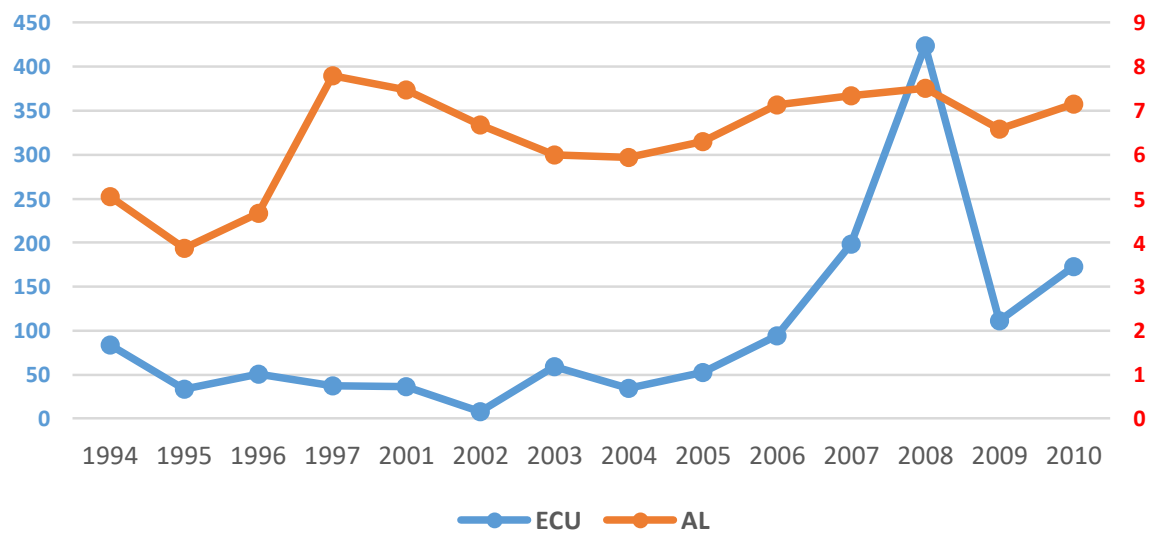

Figura 7. Razón de aplicación de patentes de no residentes/residente en la economía Fuente: Ibídem 
Ecuador al cierre del 2010 por cada patente aplicada de un residente reconocía unas 172 patentes de no residente, lo cual denota la implicación en la transferencia de divisas al exterior fruto de esta marcada desproporción. En similar período los valores para América Latina significaban que por cada patente de un residente se reconocen unas 7 patentes de no residentes.

\section{Conclusiones}

El objetivo de lograr la transformación productiva de la economía debe conducir a cambiar la condición de productores de bienes básicos, de bajo valor añadido a otros que contengan un nivel de tercerización y alto valor agregado; logrado generalmente sobre la base de la incorporación de conocimiento e innovación tecnológica. La innovación pasa a ser variable crítica en el propósito de transformar la matriz productiva de una nación propuesta en dar el salto tecnológico frente a un modelo extractivista y de manufactura de bajo perfil.

El problema mayor hoy radica en que por lo que general las economías latinoamericanas han realizado un pobre desempeño en el grado de integración de sus entramados sectoriales y ramales incorporando la variable conocimiento en una escala insuficiente, desconectada de las demandas transformadoras de sus procesos económicos, con lo cual la formación de encadenamientos productivos, aprovechando la ventaja de la innovación no ha estado en la prioridad de las agendas de cada nación.

El Ecuador ha iniciado un cambio de su política doméstica en materia de cambiar su matriz productiva, y esta variable: la innovación, ha comenzado a jugar un papel mucho más activo, para lo cual se han establecido nexos entre universidades, institutos de investigación, centros de innovación, ministerios vinculados a la producción, entidades financieras y empresas. El estudio sobre la innovación aunque siempre ha estado enfocado 
a su efecto multiplicador en la economía no ha tenido abordaje en la realidad ecuatoriana por lo que se carecen de estudios nacionales sobre el rol que está llamado a ocupar en la actualidad y perspectiva de la nación.

Con independencia de que existe una significativa elaboración desde la Secretaría Nacional de Educación Superior Ciencia y Tecnología (SENESCYT) acerca del rol del conocimiento y la innovación en el contexto ecuatoriano se carecen de evidencias empíricas que explican los grados de materialización entre el esfuerzo de la inversión en materia de conocimiento y la consecuente capacidad emprendedora que conduzca a la competitividad sostenible a escala local. 


\section{Referencias bibliográficas}

Rivera Vargas, M. Determinant factors for small business to achieve innovation, high performance and competitiveness: organizational learning and leadership style. (2014). The 6th Indonesia International Conference on Innovation, Entrepreneurship and $s$.

Alcaide, J. C., Bernúes, S., Díaz-Aroca, E., Espinosa, R., y Muñís, R. (2013). Marketing y PYMES. Las principales claves de marketing en la pequeña y mediana empresa.

Banco Interameriano de Desarrollo. (2010). Ciencia, Tecnología e Innovación en América Latina y el Caribe. New York.

BID. (Marzo de 2015). El Laberinto como América Latina y el Caribe puede navegaren la economía global. . Informe-macroeconómico-de-America-Latina-y-el-Caribe.

Botella, C., y Suárez, I. (Mayo de 2012). Innovación para el desarrollo en América Latina . Innovación para el desarrollo en América Latina. Una aproximación desde cooperación internacional. . Madrid, España : Fundación Carolina .

Braña Francisco Javier, D. R. (2016). Buen Vivir y cambio de la matriz productiva. Quito: Universidad de Cantabria.

Ciriaci, D. (2008). The role of knowledge-based supply specialisation for competitiveness: A spatial econometric approach. . Papers In Regional Science, 453-475.

Fernández, J. M. (1997). La competitividad de la empresa. Oviedo: Servicio de publicaciones Universidad de Oviedo.

Global Entrepreneurship Monitor. (2014). Informe GEM Ecuador 2014. Quito. 
Guerrieri, P. (2005). V. Technology and international competitiveness: The interdependence between manufacturing and producer services . Structural Change \& Economic Dynamics, 489-502.

Huggins, R. y Johnston, A. (2009). Knowledge Networks in an Uncompetitive Region: SME Innovation and Growth. Growth \& Change, 227-259.

Kotler, P. y Armstrong, G. (2012). Marketing. México : Pearson .

Kutchera, J., García, H., \& Fernández, A. (2014). Ëxito. Su estrategia de marketing digital en 5 pasos. México: Grupo Editorial Patria .

López, P. (2011). Aprendizaje Colaborativo para la gestión de conocimiento en redes educativas en la Web 2.0. Madrid, España .

Martín, J. R. (2016). Sobre decrecimiento y economía del bioconocimiento. Ecuador: Facultad Latinoamericana de Ciencias Sociales (FLACSO).

Martínez, E. R. (2007). Competitividad, crecimiento y capitalización de las regiones españolas . Bilbao: Fundaciín BBVA.

O.C.D.E., EUROSTAT, TRAGSA. (2005). Manual de Oslo 3ra Edición. Guía para la recogida e interpretación de datos sobre innovación. Grupo Tragsa.

Pagés, C. (2010). La era de la productividad. Cómo transformr las economías desde sus cimientos. New York : B.I.D.

Pérez, J. P., Zorrilla, D. N., \& Velázquez, R. G. (2013). Competitiveness profile and human capital in mexican companies based on innovation. (English). Revista Negotium, 109140.

Pervaiz K. Admeh, C. D. (2012). Administración de la innovación. México: Pearson.

Pervaiz, S. C. (2012). Administración de la Innovación. México: Pearson. 
Pilinkus, D., y Boguslauskas, V. (2007). New Technology Investment as a key to country competitiveness. Economics and Management, 1822-6515.

Porter, A. L., Newman, N. C., y Roessner, J. D. (2009). International high tech competitiveness: does China rank number 1? Technology Analysis \& Strategic Management, .

Priede, J., y Pereira, E. (2013). Innovation as a key factor in the international competitiveness of the European Union,. European Integration Studies., 1822-8402.

Raluka, O. (2011). The Journey to Competitiveness: EU Speeding Up on The Road paved with Knowledge and Innovation. Annals of the University of Oradea, Economic Science Series., 118-124.

Rivera Vargas, M. (2014). Determinant factors for small business to achieve innovation, high performance and competitiveness: organizational learning and leadership style.

Sánchez, R., Romero, P. y Escurra, E. (2002). La transición hacia el desarrollo sustentable. Perspectivas de América Latina y el Caribe. México : Instituto Nacional de Ecología

Santos Virgen, J. M. (2012). Las universidades de Asia Pacífico y México en la competitividad internacional. Revista Mexicana De Estudios Sobre La Cuenca Del Pacifico, 81-97.

Schepkova, I. V. (2012). Cluster policy as a mechanism to increase regional competitiveness. . Vestnik IKBFU, , 125-129.

Simmie, J., y Wood, P. (2002). Innovation and Competitive Cities in the Global Economy: Introduction to the Special Issue. European Planning Studies, 149-151. 
Macías et. Al. 2017

Spirkova, y otros. (2015). The Social Capital Influence on Enterprise Competitiveness In V4 Countries.

Torrent-Sellens, J. (2014). Emprendimiento innovador y microempresas en red. Barcelona: UOC.

World Bank Data . (2015). http://databank.worldbank.org/data/home.aspx. 